\title{
Meta-analysis of drought-induced transcriptome changes for Zea mays
}

Konstantinov D.K. ${ }^{1,2 *}$, Doroshkov A.V.., 2

${ }^{1}$ Institute of Cytology and Genetics, SB RAS, Novosibirsk, Russia

${ }^{2}$ Novosibirsk State University, Novosibirsk, Russia

*email: konstantinov@bionet.nsc.ru

Motivation: One of the major limiting factors for corn (Zea mays L.) yield is drought stress, which explains a large number of studies on this issue. However, despite all efforts, the molecular mechanisms of drought response for corn and other plants hold many unknown details. Combining the results of different research teams by transcriptome meta-analysis opens a fruitful way of searching for new genes that can clarify stress response mechanisms in the post-genomic era. Moreover, analysis of a series of transcriptomic experiments improves the accuracy of revealed relationships between environmental factors and the molecular level response. In particular, a metaanalysis of several transcriptomic datasets allows to carry out a thin border between genes that stably change expression without reference to the method of experimental modeling of drought and for genes with more flexible regulation. Method and algorithm: In this work, we perform a meta-analysis for 54 Illumina-based RNA-seq datasets found via search in NCBI SRA database by the following keywords: "drought", "dehydration", and Z. mays [organism]. We used the FastQC program for analyzing the quality of the libraries, Trimmomatic for its filtering, STAR for mapping reads to a reference genome. Function featureCounts from package subread-1.6.3-source and the R package EdgeR conducted a search for the differentially expressed genes. The meta-analysis of stressinduced transcriptomes revealed 4549 genes differentially expressed in at least one considered experiment. A significant part of them demonstrated differential expression only in some experiments. Note that a part of genes had opposite direction of expression change depending on experimental conditions. This finding revealed the relationship between the method of creating stressful conditions and the activated genetic subsystems. At the same time, 167 genes changed expression in all considered experiments. Most of them were associated with processes of hormonal signaling and transcription regulation. Obtained results can be used in the development of new strategies for maize genomic selection.

Acknowledgements: This work was funded by Russian Science Foundation grant number 19-74-10037. 